\title{
Utilização de modelos analíticos para quantifica- ção de crosstalk em WSSs
}

\author{
Daniel Alves Ribeiro Chaves 1 \\ Escola Politécnica de Pernambuco \\ Universidade de Pernambuco \\ 50.720-001 - Recife, Brasil \\ darc@ecomp.poli.br
}

\author{
Hartur Barreto Brito \\ Escola Politécnica de Pernambuco \\ Universidade de Pernambuco \\ 50.720-001 - Recife, Brasil \\ hbb@ecomp.poli.br
}

\author{
Autor 3 \\ Escola Politécnica de Pernambuco \\ Universidade de Pernambuco \\ 50.720-001 - Recife, Brasil \\ Autor2@meu-email
}

Resumo Este projeto visa estudar o efeito da interferência de crosstalk em comutadores ópticos (WSSs e OXCs) utilizando modelagem analítica e simulações monte carlo e desenvolver um programa para que esses modelos possam ser utilizados no simulator SIMTON, ajudando na análise de resultados obtidos pelo simulador.

\footnotetext{
Abstract This project tries to study the e ect of the interference and crosstalk in optical switchs (WSSs and OXCs) using analytical models and monte carlo simulation and develop a program that use these models to implement at the simulator SIMTON, helping the analysis of the results.
} 


\section{Introdução}

A disceminção da internet tem facilitado o surgimento de vários serviços de comunicação so_sticados.

A utilização de meios desses meios comunicação, como vídeo sob demanda, voz sobre IP e redes sociais, tem se tornado cada vez mais frequente. Esses serviços demandam a troca de grande quantidade de informações à elevadas taxas de transmissão. Para suportar esses serviços a infra-estrutura de telecomunica ções deve se adequar a essa requisição. Atualmente, a tecnologia que melhor atende a esses requisitos é o sistema de comunicações ópticas interligados em rede (redes ópticas). Os sistemas ópticos atuais utilizam principalmente o sistema de multiplexação por divisão de comprimento de onda ou WDM. O WDM viabiliza a transmissão simultânea de diversos_uxos informações diferentes (e.g. de usuários diferentes da rede) em uma mesma bra óptica. Cada uxo de informação é transmitido utilizando um comprimento de onda diferente.

As redes ópticas podem ser classi_cadas em: opaca, transparente e translúcida. Nas opacas, o sinal é convertido do domínio óptico para o eletrônico e novamente para o óptico (conversão O-E-O) em todos os nós da rede. Essa conversão permite a regeneração de todos os sinais que estão trafegando na rede resultando em um bom desempenho da mesma. Por outro lado seu custo de implementação é elevado, pois um grande número de dispositivos que realizam O-E-O é necessário. Nas redes transparentes não são empregadas interfaces O-E-O reduzindo seu custo de implanta ção. Porém, como não são realizadas O-E-O, há acúmulo de degradação do sinal óptico imposto pela camada física o que leva a uma perda de desempenho signi_cativa em relação às redes opacas. As redes translúcidas são redes transparentes que possuem interfaces O-E$\mathrm{O}$ (também chamadas aqui de regeneradores) estrategicamente posicionadas na rede. A rede translúcida é uma solução intermediária que procura aliar o bom desempenho da rede opaca com o baixo custo da transparente.

A utilização de uma topologia em malha. Na topologia em malha, cada nó da rede óptica pode se conectar a diversos outros nós da rede. Ou seja, um nó com essa característica possui $\mathrm{N}$ portas de entrada e $\mathrm{N}$ portas de saída. Ele deve ser capaz de comutar qualquer comprimento de onda em uma porta de entrada para qualquer porta de saída. O dispositivo que implementa essa funcionalidade é chamado na literatura de OXC (optical cross conect).

O dispositivo óptico utilizado atualmente em redes que viabiliza a construção de OXCs é o WSS (Wavelength Selective Switch). O WSS pode ser um dispositivo com uma entrada e múltiplas saídas $(1 \mathrm{xN})$, ou um dispositivo com muitas entradas e uma única saída (Nx1). O WSS pode escolher, de forma independente, quais comprimento de onda presentes em uma entrada serão transmitidos para a saída do dispositivo. As topologias de ligação mais utilizadas para se formar um OXC com WSSs são as estruturas broadcast and select (BS) e a select and select (SS). A BS apresenta um menor custo porém, em geral, introduz uma maior degradação ao sinal em comparação ao SS.

Ao ser comutado no WSS o sinal óptico pode ser degrado. Isso porque, devido a não idealidade do dispositivo, comprimentos de ondas que transportam informa ção diferentes podem interferir entre si no interior do WSS gerando uma penalidade conhecida como crosstalk. Nesse caso, a relação sinal ruído (relação que indica o quanto a intensidade de sinal é maior que a intensidade de ruído) do sinal comutado no WSS sofre uma redução pelo aumento da parcela de ruído gerado por crosstalk no interior do WSS.

Tipicamente um sinal óptico é comutado em vários nós no seu percurso. Se a cada nó de comutação há acúmulo da penalidade de crosstalk, ela pode impactar o sinal que está sendo transmitido a ponto de inviabilizar sua recepção.

\section{Formulação geral para WSS $N x 1$}

\subsection{Premissas}

O WSS considerado é mostrado na Figure 1. Ele tem N portas de entrada e 1 de saída. Há $K$ slots de frequência diferentes permitidos em cada porta de entrada/saída. Cada slot de frequência tem um comprimento de onda permitido de $\Delta \mathrm{w}$ e frequências centrais $w 1, w 2 ; \ldots, w \mathrm{~K}$. A frequência é representada pela variável independente $w$.

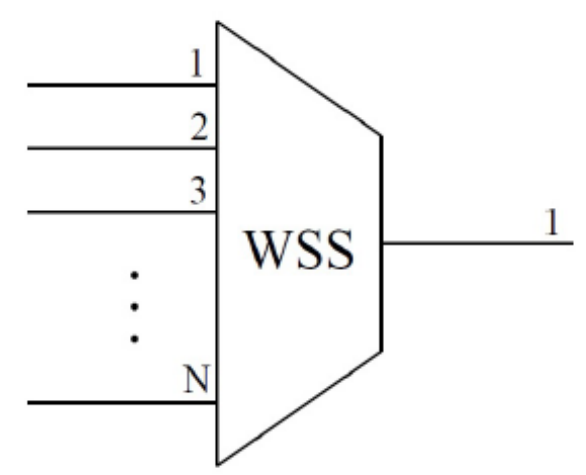

Figura 1: WSS Nx1

A matriz de comutação $\underline{\mathbf{A}}=\left\{a_{n, k}\right\}$ do WSS pode ser definida como: 


$$
a_{n, k}=\left\{\begin{aligned}
1, & \text { Se o } k \text {-th slot de frequência na } n \text {-th } \\
& \text { porta de entrada for comutada para } \\
& \text { a saída. } \\
0, & \text { Caso contrário. }
\end{aligned}\right.
$$

Considerando $g_{n}(w)$ como função transferência entre a $n-t h$ entrada do WSS e sua saída. O vetor de transferência pode ser descrito como $\underline{\mathbf{G}}(w)=$ $\left[g 1(w), \ldots, g_{n}(w)\right]^{t}$ composto por $\mathrm{N}$ funções transferência.

Suponha que na n-ésima porta de entrada do WSS exista um sinal $s_{n}(w)$ (que pode ser ou não um sinal com interferência) e um sinal adicional de ruído $r_{n}(w)$ (que pode ser gerado por diversas fontas, incluindo ruído ASE ou interferência de crosstalk). Além disso, assuma que o sinal total na n-ésima porta de entrada do WSS pode ser escrita por $s_{n}(w)+r_{n}(w)$.

\subsection{Cálculo do sinal sem interferência na sa- ída}

Algumas partes do spectrum $s_{n}(w)$ são comutadas para a saída do WSS e outras são bloqueadas. Uma forma de escrever o sinal $s^{\prime}{ }_{n}(w)$ composto apenas pelas partes do spectrum $s_{n}(w)$ que são comutadas para a saída do WSS é:

$$
s_{n}^{\prime}(w)=s_{n}(w) \sum_{k=1}^{K} a_{n, k} \pi\left(w-w_{k}\right)
$$

Sendo a função $\pi(w)$ é definida por:

$$
\pi(w)= \begin{cases}1, & \text { se }|w|<\frac{\Delta w}{2} \\ 0, & \text { Caso contrário }\end{cases}
$$

Definindo $\underline{\boldsymbol{\pi}}(w)=\left[\underline{\boldsymbol{\pi}}\left(w-w_{1}\right), \ldots, \pi\left(\mathrm{w}-w_{k}\right)\right]^{t}$, e $\underline{\mathbf{S}^{\prime}}=$ $\left[s^{\prime} 1(w), \ldots, s^{\prime}{ }_{N}(w)\right]^{T}$ pode-se escrever (2) na forma de matriz como:

$$
\underline{\mathbf{S}}^{\prime}(w)=(\underline{\mathbf{A}} \cdot \underline{\mathbf{\Pi}}(w)) \otimes \underline{\mathbf{S}}(w)
$$

Sendo a representação do produto de matrizes de Hadamard. Dadas duas matrizes $\underline{\mathbf{A}}=\left\{a_{i, j}\right\}$ e $\underline{\mathbf{B}}=\left\{b_{i, j}\right\}$ o produto de Hadamard retorna a matriz $\underline{\mathbf{C}}=\left\{c_{i, j}\right\}$ considerando que $c_{i, j}=a_{i, j} b_{i, j}$.
O crosstalk em um WSS é gerado pela imperfeição no bloqueio do spectrum de frequência que não deveria ser comutado do n-th porta de entrada para a porta de saída. Observe que $s_{n}^{\prime}($ w) contém uma porção do spectrum de frequência que é comutado da entrada para saída e não sofre nenhum bloqueio. Assim, o sinal na saída que não recebe interferência de $r_{n}^{\prime}(\mathrm{w})$ nem do crosstalk gerado pelo WSS pode ser calculado como:

$$
y^{S}(w)=\sum_{n=1}^{N} g_{n}(w) s_{n}^{\prime}(w)
$$

Ou em notação de matriz:

$$
y^{S}(w)=\underline{\mathbf{G}}^{T}(w) \cdot \underline{\mathbf{S}}^{\prime}(w)=\underline{\mathbf{G}}^{T}(w) \cdot[(\underline{\mathbf{A}} \cdot \underline{\mathbf{I}}(w)) \otimes \underline{\mathbf{S}}(w)]
$$

\subsection{Cálculo do sinal com interferência na sa- ída}

O sinal total na entrada (sinal mais sinal de ruído) é $\mathrm{sn}(w)+r_{n}(w)$. Assim, o sinal de saída com interferência gerada pelo crosstalk do WSS e ruído das portas de entrada podem ser cálculadas por:

$$
y^{\text {total }}(w)=\sum_{n=1}^{N} g_{n}(w)\left[s_{n}(w)+r_{n}(w)\right]
$$

Que pode ser escrito na notação de matriz como:

$$
y^{\text {total }}(w)=\underline{\mathbf{G}}^{T}(w) \cdot[\underline{\mathbf{S}}(w)+\underline{\mathbf{R}}(w)]
$$

Sendo $\underline{\mathbf{R}}(w)=\left[r_{1}(w), \ldots, r_{N}(w)\right]^{T}$.

\subsection{Ruído saída e interferência de cosstalk to- tal}

Como foi descrito anteriormente $y^{\text {total }}(w) \mathrm{e}$ $y^{s}(w)$ são, respectivamente, o sinal com interferência e sem interferência de ruído e crosstalk. Portanto, o total de ruído e interferência de crosstalk $y^{I}(w)$ verificado na saída do WSS pode ser calculada como:

$$
y^{I}(w)=y^{\text {total }}(w)-y^{s}(w)
$$

Assim, 


$$
y^{I}(w)=\underline{\mathbf{G}}^{T}(w)[\underline{\mathbf{S}}(w)+\underline{\mathbf{R}}(w)-(\underline{\mathbf{A}} \cdot \underline{\mathbf{\Pi}}(w)) \otimes \underline{\mathbf{S}}(w)]
$$

Resumindo:

$$
\begin{aligned}
& y^{S}(w)=\underline{\mathbf{G}}^{T}(w) \cdot[(\underline{\mathbf{A}} \cdot \underline{\mathbf{\Pi}}(w)) \otimes \underline{\mathbf{S}}(w)] . \\
& y^{I}(w)=\underline{\mathbf{G}}^{T}(w) \cdot[\underline{\mathbf{S}}(w)+\underline{\mathbf{R}}(w)-(\underline{\mathbf{A}} \cdot \underline{\mathbf{I}}(w)) \otimes \underline{\mathbf{S}}(w)] \quad \text { (Noise and Xtalk) }
\end{aligned}
$$

\section{Formulação Geral para WSS}

\subsection{Premissas}

O WSS considerado está representado na Figure 2. O WSS considerado tem 1 porta de entrada e $\mathrm{N}$ portas de saída. Há K slots de frequência permitidos em cada porta de entrada/saída. Cada slot de frequência tem um comprimento de onda permitido de $\Delta \mathrm{w}$ e frequências centrais de $w 1 ; w 2 ;::: ; w k$. A frequência é representada pela variável independente $w$

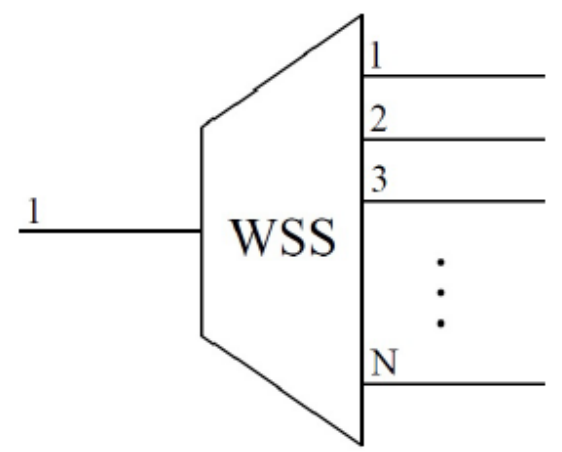

Figura 2: WSS N1xN

A matriz de comutação do WSS $\underline{\mathbf{B}}=\left\{b_{n, k}\right\}$ é definida por:

Considere $f_{n}(w)$ como sendo função transferência entre a entrada e a $n$-th saída do WSS. $\mathrm{O}$ vetor de transferência pode ser descrito como $\underline{\mathbf{F}}(w)=\left[f_{1}(w), \ldots, f_{N}(w)\right]^{T}$ composto por $N$ funções-transferência.

Suponha que na porta de entrada do WSS há um sinal principal $s(w)$ (que pode ou não conter interferência) e um sinal adicional de ruído $r(w)$ (que pode ser oriundo de diferentes fontes incluindo ruído ASE ou interferência por crosstalk). Além disso, assuma que o sinal total da porta de entrada do WSS pode ser escrito como $s(w)+r(w)$.

\subsection{Cálculo do sinal sem interferência na sa- ída}

Algumas partes do spectrum $s(w)$ são comutadas para a saída do WSS e a outras são bloqueadas. Uma forma de escrever o sinal $s^{\prime}{ }_{n}(w)$ composto apenas pelas partes do spectrum $s(w)$ que são comutadas para $n$-th saída do WSS é:

$$
s_{n}^{\prime}(w)=s(w) \sum_{k=1}^{K} b_{n, k} \pi\left(w-w_{k}\right)
$$

Sendo a função $\pi(w)$ definida por (3). Definindo $\underline{\Pi}(w)=\left[\pi\left(w-w_{1}\right), \ldots, \pi\left(w-w_{K}\right)\right]^{T}$ e $\underline{\mathbf{S}}^{\prime}(w)=$ $\left[s_{1}^{\prime}(w), \ldots, s_{N}^{\prime}(w)\right]^{T}$ pode-se escrever (13) utilizando notação de matriz como:

$$
\underline{\mathbf{S}}^{\prime}(w)=[\underline{\mathbf{B}} \cdot \underline{\Pi}(w)] s(w)
$$

O crosstalk em um WSS é gerado pela imperfeição no bloqueio do spectrum de frequência que não deveria ser comutado da porta de entrada para a $n$-th porta de saída. Observe que $s_{n}^{\prime}(w)$ contém uma porção do spectrum de frequência que é comutado da entrada para a $n$-th saída e não sofre nenhum bloqueio. Assim, o sinal $y_{n}^{S}(w)$ na $n$-th porta de saída que não recebe interferência de $r(w)$ nem do crosstalk gerado pelo WSS pode ser calculado como:

$$
y_{n}^{S}(w)=f_{n}(w) s_{n}^{\prime}(w)
$$

Definindo $\underline{\mathbf{Y}}^{S}(w)=\left[y_{1}^{S}(w), \ldots, y_{N}^{S}(w)\right]^{T}$ pode-se escrever (15) em notação de matriz como:

$\underline{\mathbf{Y}}^{S}(w)=\underline{\mathbf{F}}(w) \otimes \underline{\mathbf{S}}^{\prime}(w)=s(w) \underline{\mathbf{F}}(w) \otimes[(\underline{\mathbf{B}} \cdot \underline{\boldsymbol{\Pi}}(w))]$

Note que $s(w)$ é um escalar. Então pode ser colocado à esquerda da equação.

\subsection{Cálculo da interferência do sina na saída}

O sinal total de entrada (sinal mais ruído é dado por $s(w)+r(w)$. Assim, o sinal de interferência gerada pelo crosstalk do WSS e o sinal de ruído da porta de entrada do WSS pode ser calculado por:

$$
y_{n}^{\text {total }}(w)=[s(w)+r(w)] f_{n}(w)
$$


Que pode ser escrito na notação de matriz como:

$$
\underline{\mathbf{Y}}^{\text {total }}(w)=\underline{\mathbf{F}}(w)[s(w)+r(w)]
$$

Sendo $\underline{\mathbf{Y}}^{\text {total }}(w)=\left[y_{1}^{\text {total }}(w), \ldots, y_{N}^{\text {total }}(w)\right]^{T}$.

\subsection{Total de ruído na saída e interferência de crosstalk}

Como foi discutido, $y_{n}^{\text {total }}(w)$ e $y_{n}^{S}(w)$ são, respectivamente, os sinais da $n$ -th porta de saída com e sem interferência gerada pelo ruído e crosstalk $y_{n}^{I}(w)$ verificada na $n$-th porta de saída do WSS pode ser calculada por:

$$
y_{n}^{I}(w)=y_{n}^{\text {total }}(w)-y_{n}^{s}(w)
$$

Que pode ser escrita em notação de matriz como:

$\underline{\mathbf{Y}}^{I}(w)=\underline{\mathbf{Y}}^{\text {total }}(w)-\underline{\mathbf{Y}}^{s}(w)=\underline{\mathbf{F}}(w)[s(w)+r(w)]-s(w)$

Que pode ser reescrita como:

$$
\underline{\mathbf{Y}}^{I}(w)=s(w)[\underline{\mathbf{F}}(w)-\underline{\mathbf{F}}(w) \otimes(\underline{\mathbf{B}} \cdot \underline{\mathbf{\Pi}}(w))]+r(w) \underline{\mathbf{F}}(w)
$$

Resumindo:

$$
\begin{aligned}
& \underline{\mathbf{Y}}^{S}(w)=s(w) \underline{\mathbf{F}}(w) \otimes[(\underline{\mathbf{B}} \cdot \underline{\mathbf{I}}(w))] . \\
& \left.\underline{\mathbf{Y}}^{I}(w)=s(w) \underline{\mathbf{F}}(w)-\underline{\mathbf{F}}(w) \otimes(\underline{\mathbf{B}} \cdot \underline{\mathbf{I}}(w))\right]+r(w) \underline{\mathbf{F}}(w) \text { (Noise and Xtalk) }
\end{aligned}
$$

$(22)$

\section{Formulação para arquitetura de nó broadcast and select}

\subsection{Premissas}

Nesta seção serão aplicados os resultados obtidos em (11) para estabelecer o modelo analítico para o nó com arquitetura broadcast and select. Essa arquitetura é mostrada na Figure 3. No nó há $N$ fibras de entrada são indexadas com $i$ enquanto as fibras de saída são indexadas $\operatorname{com} j$.

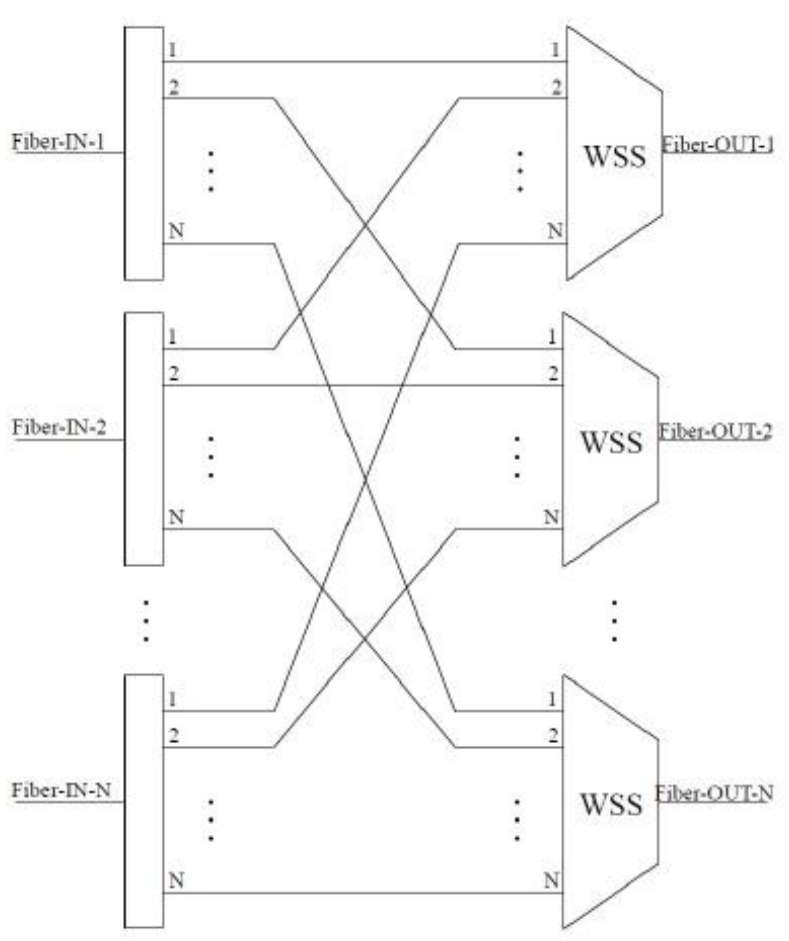

Figura 3: Arquitetura de nó broadcast and select

Suponha que, na $i$-th fibra da porta de entrada, há um sinal principal $s_{i}^{f b r}(w)$ (que pode ou não ser um sinal principal com interferência) e um sinal adicional de ruído $r_{i}^{f b r}(w)$ (que pode incluir várias fontes diferentes como o ruído ASE ou interferência de crosstalk). Além disso, assuma que o sinal total na $i$-th porta de entrada pode ser escrita como $s_{i}^{f b r}(w)+r_{i}^{f b r}(w)$. Considere que o splitter localizado na $i$-th fibra de entrada tem uma perda de $l_{i}^{s p}$.

\subsection{Formulação}

O sinal $y_{j}^{S}(w)$ na $j$-th saída do WSS pode ser descrito em função de um vetor de transferência $\boldsymbol{G}_{j}^{T}(w)$, uma matriz de comutação $\underline{A}_{j}$, um vetor de perdas $\boldsymbol{L}^{s p}$ e um vetor de sinais $\underline{S^{f b r}}$ da seguinte forma:

$y_{j}^{S}(w)=\frac{1}{N} \underline{\mathbf{G}}_{j}^{T}(w) \cdot\left\{\left[\underline{\mathbf{A}}_{j} \cdot \underline{\mathbf{\Pi}}(w)\right] \otimes\left[\underline{\mathbf{L}}^{s p} \otimes \underline{\mathbf{S}}^{f b r}\right]\right\}$ 
O ruído e o crosstalk podem ser descritos em função da quantidade $N$ de portas do WSS, uma matriz de comutação $\underline{\mathbf{A}}_{j}$, um vetor de perdas $\underline{\mathbf{L}}^{s p}$ e o vetor de ruído adicional $\underline{\mathbf{R}}^{f b r}$ da seguinte forma:

$$
y_{j}^{I}(w)=\frac{1}{N} \underline{\mathbf{G}}_{j}^{T}(w) \cdot\left[\underline{\mathbf{L}}^{s p} \otimes \underline{\mathbf{S}}^{f b r}+\underline{\mathbf{L}}^{s p} \otimes \underline{\mathbf{R}}^{f b r}-\left(\underline{\mathbf{A}}_{j} \cdot \underline{\mathbf{I}}(w)\right) \otimes\left(\underline{\mathbf{L}}^{s p} \otimes \underline{\mathbf{S}}^{f b r}\right)\right]
$$

\section{Formulação para arquitetura de nó switch and select}

Nesta seção serão aplicados os resultados obtidos em (11) e (22) para estabelecer o modelo analítico para o nó com arquitetura switch and select. Essa arquitetura é mostrada na Figure 3. No nó há $\mathrm{N}$ bras de entrada e $\mathrm{N}$ bras de saída. As bras de entrada são indexadas com $\overline{\mathrm{i}}$ enquanto as de saída são indexadas com j.

Pode-se calcular o sinal do $j$-th WSS Nx1 em função de uma matriz de comutação $\underline{\mathbf{A}}_{j}$ e pelas matrizes $\underline{\mathbf{M}}^{T}$ e $\mathbf{C}^{T}$ que podem ser calculadas, respectivamente, por $\underline{\mathbf{M}}^{T} \cdot \underline{\mathbf{E}}_{j}$ e $\underline{\mathbf{C}}^{T} \cdot \underline{\mathbf{E}}_{j}$, como:

$$
y_{j}^{S}(w)=\underline{\mathbf{G}}_{j}^{T}(w)\left[\left(\underline{\mathbf{A}}_{j} \cdot \underline{\mathbf{\Pi}}(w)\right) \otimes\left(\underline{\mathbf{M}}^{T} \cdot \underline{\mathbf{E}}_{j}\right)\right]
$$

E a interferência pode ser calculada como:

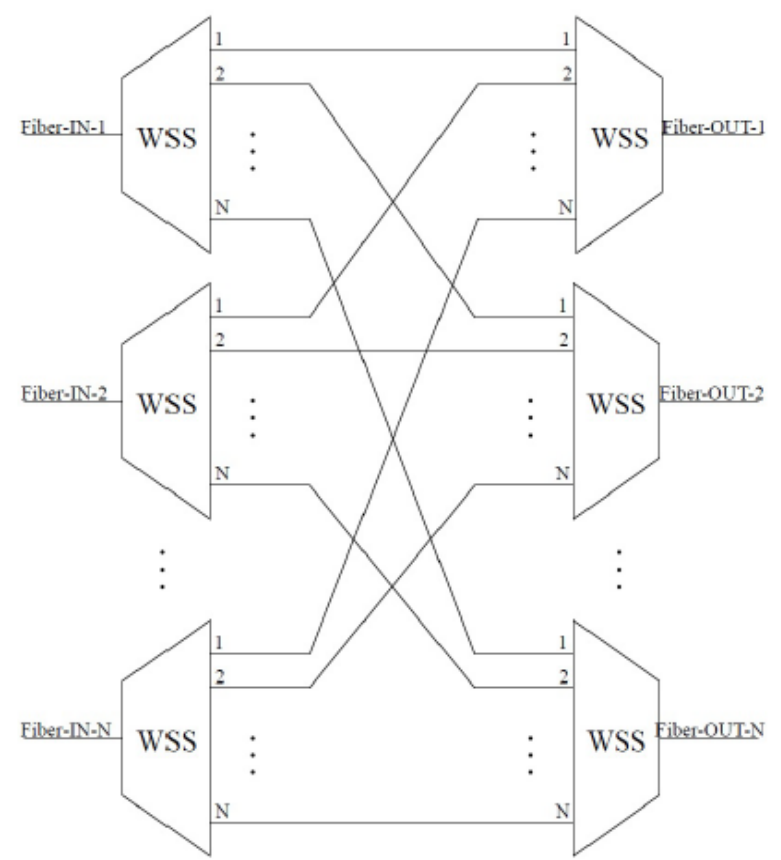

Figura 4: Arquitetura de nó switch and select

$$
y_{j}^{S}(w)=\underline{\mathbf{G}}_{j}^{T}(w)\left[\underline{\mathbf{M}}^{T} \cdot \underline{\mathbf{E}}_{j}+\underline{\mathbf{C}}^{T} \cdot \underline{\mathbf{E}}_{j}-\left(\underline{\mathbf{A}}_{j} \cdot \underline{\mathbf{\Pi}}(w)\right) \otimes\left(\underline{\mathbf{M}}^{T} \cdot \underline{\mathbf{E}}_{j}\right)\right]
$$

\section{Implementação da Modelagem}

Esta seção irá comentar a respeito do programa desenvolvido para ser utilizado em simulações.

\subsection{Premissas}

Para representação dos valores foram utilizados tabelas csv. Entre elas algumas devem ser preenchidas antes da execução do programa, são elas: Tabela com sianis de entrada dos WSSs, tabelas referentes 6 às funções transferência do primeiro e segundo layer e tabela referente a topologia do nó.

A partir dessas tabelas o programa é capaz de calcular os sianis de saída do layer 1 e alocar, de acordo com a topologia, como sinal de entrada do layer 2 para que possa ser calculado o sinal de saída do layer 2 .

Com esses resultados é possível retirar informações sobre o ruído de acordo com a modelagem demonstrada nas seções anteriores. 